\title{
Automatic Atlas-Guided Constrained Random Walker Algorithm for 3D Segmentation of Muscles on Water Magnetic Resonance Images
}

\author{
Faezeh Fallah and Bin Yang \\ Institute of Signal Processing \\ and System Theory, University of Stuttgart \\ Email: \{faezeh.fallah, bin.yang\}@iss.uni-stuttgart.de
}

\author{
Fabian Bamberg \\ Department of Diagnostic and Interventional \\ Radiology, University Clinic of Tübingen \\ Email: fabian.bamberg@med.uni-tuebingen.de
}

\begin{abstract}
Automatic segmentation of distinct muscles is a crucial step for quantitative analysis of muscle's tissue properties. Magnetic resonance (MR) imaging provides a superior soft tissue contrast and noninvasive means for assessing muscular characteristics. However, automatic segmentation of muscles using common morphological MR imaging is very challenging as the intensities and textures of adjacent muscles are similar and the boundaries between them are mostly invisible or discontinuous. In this paper, we propose a novel fully automatic framework for 3D segmentation of muscles on water MR images. This framework generates the 3D average and probabilistic atlases of the targeted muscle to automatically define the labeled seeds, the edges weights, and the constraints of a constrained Random Walker algorithm. Also, the low-pass filtered atlas-derived muscle probability map is used to augment the intensities prior to the graph-based segmentation. This enables automatic localization of the targeted muscle and enforces dissimilarities between its intensities and the intensities of adjacent lean tissues. The proposed algorithm outperforms the original random Walker algorithm and the conventional multi-atlas registration for muscle segmentation and is less sensitive to errors in the manually segmented muscle masks used for training (atlas computation).
\end{abstract}

\section{INTRODUCTION}

Automatic muscle segmentation is a crucial step for quantitative analysis of muscle's tissue properties such as its mass, fiber type composition, and morphometrics reflecting overall muscle quality. Those analyses are proven to be necessary for early diagnosis and efficient treatment of several muscular disorders [1]. Magnetic resonance (MR) imaging provides a superior soft tissue contrast and noninvasive means for quantifying tissue properties. However, in MR images acquired by common morphological imaging techniques, most lean tissues manifest similar intensities and textures. Also, common spatial resolutions of MR imaging lead to a weak, discontinuous, or invisible boundary between adjacent tissues. This hinders development of fully automatic segmentation algorithms based on MR images.

Graph-based Random Walker algorithm for segmentation [2] can handle noisy, weak, non-convex, and discontinuous boundaries between adjacent tissues. However, this supervised segmentation approach demands user interaction to define labeled seeds for different classes and has difficulties in differentiating objects of similar intensities or textures. Moreover, its performance depends on the number and distribution of the defined seeds [2]-[4].

To address these issues, some varieties of the Random
Walker algorithm have been proposed to incorporate prior knowledge about object's shape and adjacency into the image graph [3]-[5]. Those priors can be inferred by using learningbased algorithms such as multi-atlas registration [6], [7] or statistical shape and local appearance models [6], [8]. However, they demand a comprehensive training data to capture a sufficient range of shape and intensity variations.

Such training data are typically generated from manually segmented masks of the targeted object on several image data sets. Manual segmentation is tedious, costly, error-prone, and subject to inter- and intra-evaluator variabilities. These hinder the computation of an accurate prior model when a sufficient amount of expertise, time, cost, or image data set is not available. Furthermore, the accuracy of the manually segmented volumes are limited by the quality of the used images. Thus manual segmentation is essentially affected by the same errors that impact automatic segmentation.

In this paper, we propose a fully automatic algorithm for 3D segmentation of the distinct muscles on water MR images by addressing the aforementioned challenges. This algorithm employes a constrained Random Walker algorithm preceded by computing muscle's average and probabilistic atlases.

The atlas-derived muscle probability map is used to automatically localize the targeted muscle and to enforce dissimilarities between muscle's intensities and the intensities of adjacent tissues. Also, this map and the detected boundaries between tissues define the labeled seeds, the edges' weights, and the constraints of the constrained Random Walker algorithm to improve muscle probability map for an accurate muscle segmentation on non-contrast-enhanced MR images.

For segmentation, muscle probability maps are generated by a conventional multi-atlas registration and its combination with the original Random Walker algorithm [2] or the proposed constrained Random Walker algorithm. For each subject's image, these three sets of probability maps are thresholded to obtain the automatically segmented muscle masks. These masks are evaluated against the manually obtained ground truths. By introducing errors in the manually segmented muscle masks used for atlas computation, the sensitivities of the above methods to these errors are also evaluated.

These evaluations are done for automatic 3D segmentation of the psoas major and quadratus lumborum muscles on water MR images. However, all the compared methods are generic and applicable to other muscle compartments as well. 


\section{MATERiALS AND METHODS}

\section{A. Preprocessing of MR Images}

The proposed method is developed using 3D water MR images of 48 (20 training and 28 test) asymptomatic volunteers. These images are acquired by a chemical-shift-encoded pulse sequence with an isotropic spatial resolution of $1.7 \mathrm{~mm}$. In these images, lean tissues are bright and fatty compartments are suppressed. Each $3 \mathrm{D}$ image is normalized in intensity to the range [0,1]. All 3D images are resized to a common size and the edges between different tissues are detected using a 3D Canny edge detector [9]. The detected edges are used in the new constrained Random Walker algorithm described in subsection II-C. Fig. 1 shows an axial slice of a 3D water image with the targeted muscles and the detected edges.

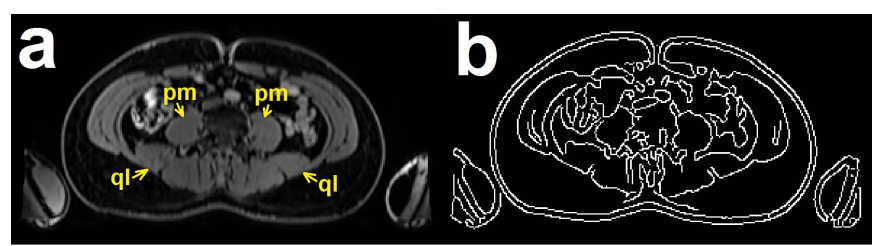

Fig. 1. An axial slice of a 3D water image showing the psoas major (pm) and quadratus lumborum (ql) muscles (a), and the detected edges (b).

\section{B. The 3D Average and Probabilistic Muscle Atlases}

The 3D average and probabilistic muscle atlases are computed using the training data set. This data set consists of the 3D water images and the manually segmented muscle masks of $M=20$ volunteers. These masks are denoted by $\left\{M_{k}\right\}_{k=1}^{M}$.

The 3D water image of an arbitrarily selected volunteer is taken as a source and is rigidly registered to $M-1$ remaining water images using a block matching procedure [10]. The $M-1$ deformed images $\left\{D_{k}^{(0)}\right\}_{k=2}^{M}$ and the source image $D_{1}^{(0)}$ are averaged to yield the first 3D average atlas $A^{(0)}$. The resulting transformations are denoted by $\left\{T_{k}^{r}\right\}_{k=1}^{M}$, where $T_{1}^{r}$ is an identity transform for the source image.

To reduce the computational burden of the subsequent nonrigid registrations, a resolution pyramid of $L$ levels is built from $\left\{D_{k}^{(0)}\right\}_{k=1}^{M}$. In the $l^{\text {th }}$ level, $\left\{D_{k}^{(l, 0)}\right\}_{k=1}^{M}$ is obtained by down-sampling $\left\{D_{k}^{(0)}\right\}_{k=1}^{M}$ to the spatial resolution of this level. The lowest (highest) level corresponds to the coarsest (finest) spatial resolution. The computations start from the coarsest resolution $l=1$, in which $A^{(l, 0)}$ is obtained by downsampling $A^{(0)}$ to the coarsest resolution.

In the $l^{\text {th }}$ level, over multiple iterations $\{i\}_{i=1}^{I_{l}}$ of a non-rigid phase-difference-based Morphon registration algorithm [11], $\left\{D_{k}^{(l, 0)}\right\}_{k=1}^{M}$ are registered to $A^{(l, 0)}$. The deformation fields of the $i^{\text {th }}$ iteration $\left\{T_{k}^{(l, i)}\right\}_{k=1}^{M}$ get updated using a diffeomorphic field accumulation [12]. The final (accumulated) deformation fields of the $l^{\text {th }}$ level $\left\{T_{k}^{\left(l, I_{l}\right)}\right\}_{k=1}^{M}$ yields the warped images as $\left\{D_{k}^{\left(l, I_{l}\right)}\right\}_{k=1}^{M}=\left\{T_{k}^{\left(l, I_{l}\right)}\left(D_{k}^{(l, 0)}\right)\right\}_{k=1}^{M}$. Then the average atlas of this level is updated by $A^{(l)}=\frac{1}{M} \sum_{k=1}^{M} D_{k}^{\left(l, I_{l}\right)}$. A spline interpolation of $A^{(l)}$ yields $A^{(l+1,0)}$ for the next level.

The above procedure is repeated until the average atlas $A^{(L)}$ and the accumulated deformation fields $\left\{T_{k}^{\left(L, I_{L}\right)}\right\}_{k=1}^{M}$ of the finest (original) resolution are obtained. In our case, $L=5$, $I_{L}=1$, and $\left\{I_{l}=(L-l) * L\right\}_{l=1}^{L-1}$.

The overall transformations $\left\{T_{k}=T_{k}^{r} \text { o } T_{k}^{\left(L, I_{L}\right)}\right\}_{k=1}^{M}$ are used to warp $\left\{M_{k}\right\}_{k=1}^{M}$ yielding $\left\{T_{k}\left(M_{k}\right)\right\}_{k=1}^{M}$. These warped binary masks are overlaid and their overlap percentages are counted to produce the 3D probabilistic muscle atlas $P$. This way, $P_{p m}$ for the psoas major (pm) and $P_{q l}$ for the quadratus lumborum (ql) muscle are obtained separately. Regarding the disconnection of these muscles, an overlay of $P_{p m}$ and $P_{q l}$ is denoted by $P=P_{p m}+P_{q l}$. Fig. 2 shows an axial slice of $A^{(0)}, A^{(L)}$, and $P=P_{p m}+P_{q l}$. These atlases are used by the new constrained Random walker algorithm.

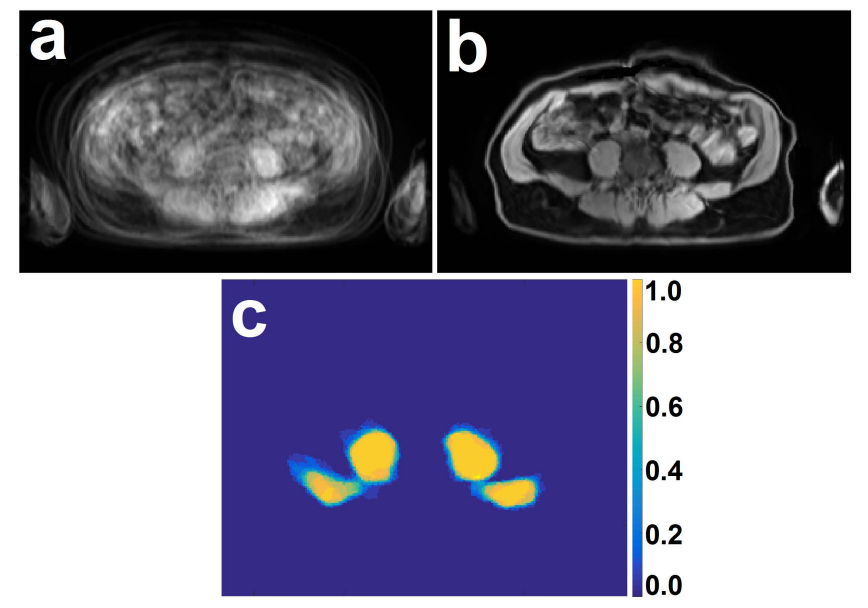

Fig. 2. An axial slice of $A^{(0)}$ (a), $A^{(L)}$ (b), and $P=P_{p m}+P_{q l}$ (c).

\section{The Constrained Random Walker Algorithm}

The Random Walker algorithm [2] models an image as a graph $\mathcal{G}=\langle\mathcal{V}, \mathcal{E}\rangle$, where each vertex $v_{i} \in \mathcal{V}$ represents a voxel and spatially neighboring vertices are connected with undirected edges $e_{i j} \in \mathcal{E}$. The degree $d_{i}$ of a vertex $v_{i}$ is given by $d_{i}=\sum_{e_{i j} \in \mathcal{E}} w_{i j}^{r w}$, where $w_{i j}^{r w}$ is the weight of $e_{i j}$. We consider a 26 -connected graph stemming from a $3 \times 3 \times 3$ stencil of second order neighborhoods. For binary voxel classification, this algorithm uses a set of foreground $S_{F}$ and a set of background $S_{B}$ seeds with $S_{F} \subset \mathcal{V}, S_{B} \subset \mathcal{V}$ and $S_{F} \cap S_{B}=0$ to determine the probability $0 \leq p_{i}^{r w} \leq 1$ that a random walker starting from $v_{i}$ will first reach one of the foreground seeds before arriving at any of the background seeds. Clearly, $\forall v_{i} \in S_{F}, p_{i}^{r w}=1$ and $\forall v_{i} \in S_{B}, p_{i}^{r w}=0$. Also, $\forall v_{i} \in \mathcal{V}-\left(S_{F} \cup S_{B}\right)$ the following condition holds:

$$
p_{i}^{r w}=\frac{1}{d_{i}} \sum_{e_{i j} \in \mathcal{E}} w_{i j}^{r w} \cdot p_{j}^{r w} .
$$

Solving the above system of linear equations returns the probability $p_{i}^{r w}$ that vertex $v_{i} \in \mathcal{V}-\left(S_{F} \cup S_{B}\right)$ has label $S_{F}$. Then the foreground object (targeted muscle) is segmented by $p_{i}^{r w} \geq 0.5$. The above system of equations can be extended by imposing additional constraints on the set of voxels located on the boundaries between different tissues $\left(S_{H}\right)$ and the set of voxels located around these boundaries $\left(S_{S}\right)$. The resulting constrained Random Walker algorithm computes the probability $p_{i}^{c r w}$ that vertex $v_{i}$ has label $S_{F}$ as [4]:

$$
\begin{array}{r}
p_{i}^{c r w}=\underset{p_{i}}{\arg \min }\left(\sum_{e_{i j} \in \mathcal{E}} w_{i j}^{c r w} \cdot\left(p_{i}-p_{j}^{c r w}\right)^{2}\right. \\
\left.\quad+\sum_{v_{i} \in S_{S}} \lambda_{i} \cdot\left(p_{i}-0.5\right)^{2}\right), \\
\text { s.t. }\left\{\begin{array}{ll}
p_{i}^{c r w}=1, & v_{i} \in S_{F} \\
p_{i}^{c r w}=0, & v_{i} \in S_{B} \\
p_{i}^{c r w}=0.5, & v_{i} \in S_{H}
\end{array} .\right.
\end{array}
$$


Vanishing the derivative of the above cost function with respect to $p_{i}$ yields the following system of equations that can be solved efficiently [4]:

$p_{i}^{c r w}=\left\{\begin{array}{l}\frac{1}{d_{i}+\lambda_{i}}\left(\sum_{e_{i j} \in \mathcal{E}} w_{i j}^{c r w} \cdot p_{j}^{c r w}+0.5 \lambda_{i}\right), v_{i} \in S_{S} \\ \frac{1}{d_{i}}\left(\sum_{e_{i j} \in \mathcal{E}} w_{i j}^{c r w} \cdot p_{j}^{c r w}\right), \quad v_{i} \in \mathcal{V}-S_{C}\end{array}\right.$

with $S_{C}=S_{F} \cup S_{B} \cup S_{H} \cup S_{S}$.

In our approach, we do not set $\lambda_{i}$ empirically constant, rather equal to $\lambda_{i}=\frac{1}{\left|g_{i}^{s}-\overline{g^{h}}\right|}$, where $g_{i}^{s}$ is the intensity of $v_{i} \in S_{S}$ and $\overline{g^{h}}$ is the average intensity of all $v_{i} \in S_{H}$. This yields a spatially varying regularizer that weighs the contribution of $v_{i} \in S_{S}$ according to the similarity of its intensity to $\overline{g^{h}}$.

Eq. 3 implies addition of a virtual neighbor vertex of 0.5 probability to each $v_{i} \in S_{S}$ via a virtual edge with weight $\lambda_{i}$.

In addition to the spatially varying regularization, our main contributions to the constrained Random Walker algorithm, in difference to the previous works [2], [4], are the ways of

- determining $S_{F}, S_{B}, S_{H}$, and $S_{S}$,

- defining edge weights of the Random walker graph.

D. Determination of $S_{F}, S_{B}, S_{H}$, and $S_{S}$

The average atlas $A^{(L)}$ (obtained from the training phase) is warped to the $3 \mathrm{D}$ water image of each test subject by using the aforementioned rigid and non-rigid registrations [10], [11]. The resulting deformation fields of those registrations are used to warp the muscle probabilistic atlas $P$. This yields an atlasderived probability map $P^{m a r}$ for each test subject (mar stands for the multi-atlas registration). It contains the probability $p_{i}^{\text {mar }}$ of each $v_{i} \in \mathcal{V}$ to be part of the targeted muscle. Fig. 3 shows an axial slice of the warped $A^{(L)}$ and $P=\left(P_{p m}+P_{q l}\right)$, and the overlaid probability maps $P^{\text {mar }}=\left(P_{p m}^{\text {mar }}+P_{q l}^{\text {mar }}\right)$.
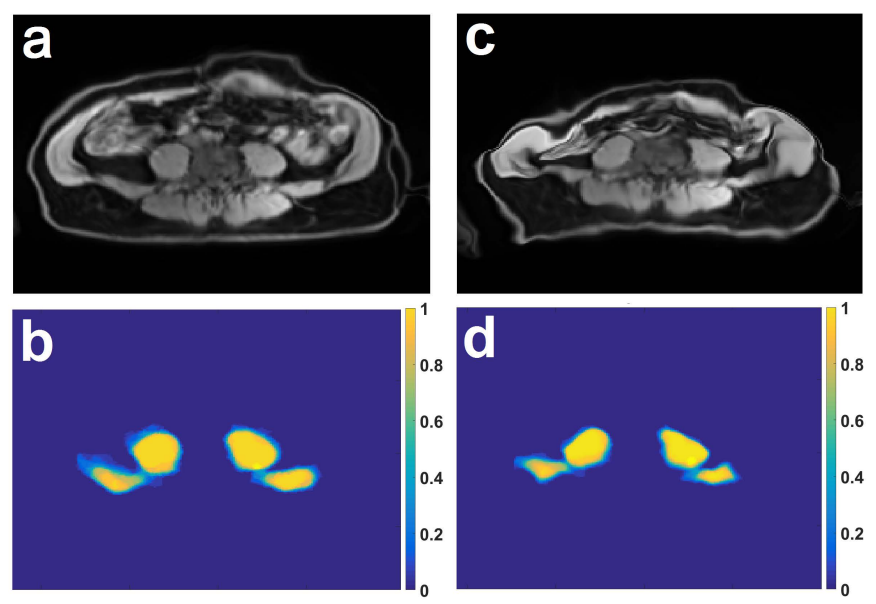

Fig. 3. An axial slice of: a) the rigidly warped $A^{(L)}$, b) the rigidly warped $\left.P=\left(P_{p m}+P_{q l}\right), \mathrm{c}\right)$ the non-rigidly warped version of (a), d) the nonrigidly warped version of (b) or the atlas-derived muscle probability map, i.e., $P^{\text {mar }}=\left(P_{p m}^{\text {mar }}+P_{q l}^{\text {mar }}\right)$.

To extract the labeled seeds of the constrained random walker algorithm, the $3 \mathrm{D}$ atlas-derived muscle probability map $P^{m a r}$ is spatially low-pass filtered by a Gaussian kernel with a standard deviation $\mathrm{SD}=3$ (see subsection II-F).

The final segmentation is based on the probability map $P^{c r w}$ computed by the constrained random walker algorithm. Thus, the above filtering does not blur the final segmentation. It rather reduces the impact of the low confident regions of $P^{\text {mar }}$. These regions are mostly located near the edges of the targeted muscle. They stem from a small number of training samples or errors in the manually segmented muscle masks $\left\{M_{k}\right\}_{k=1}^{M}$ used for atlas computation. In the low-pass filtered map $\widehat{P}^{m a r}$, regions of $\hat{p}_{i}^{\text {mar }} \geq 0.9$ and $\hat{p}_{i}^{\text {mar }} \leq 0.1$ are assigned to $S_{F}$ and $S_{B}$, respectively. $S_{H}$ and $S_{S}$ are given by

$$
\begin{aligned}
& S_{H}=E \cap\left(\mathcal{V}-\left(S_{F} \cup S_{B}\right)\right), \\
& S_{S}^{\prime}=\left\{v_{i}|| \hat{p}_{i}^{\text {mar }}-0.5 \mid \leq \epsilon\right\} \cap\left(\mathcal{V}-\left(S_{F} \cup S_{B}\right)\right), \\
& S_{S}=S_{S}^{\prime}-S_{H},
\end{aligned}
$$

where $E$ is the set of voxels on the detected boundary edges (see subsection II-A) and $\epsilon=0.03$ is an empirically set threshold. The above definitions ensure no overlap between $S_{F}, S_{B}, S_{H}$, and $S_{S}$. Fig. 4 shows $S_{F}, S_{B}, S_{S}^{\prime}$, and $S_{H}$ for an axial slice of the water image of a test subject.

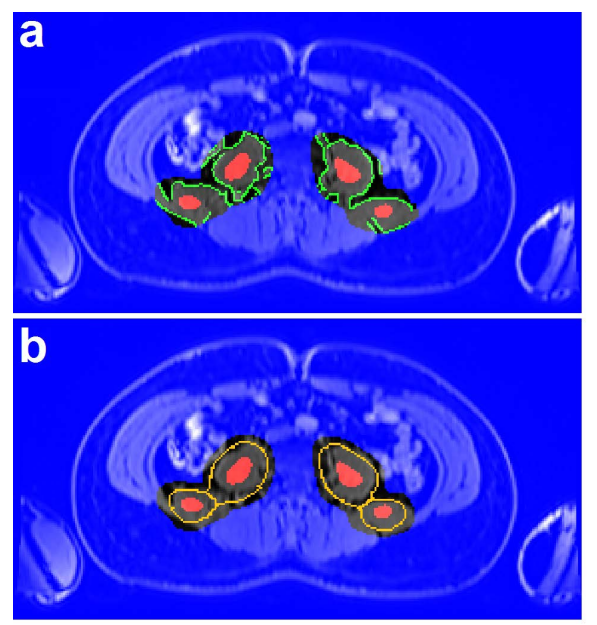

Fig. 4. a) The regions of $S_{B}$ (blue), $S_{F}$ (red), and $S_{H}$ (green) for an axial slice of the water image of a test subject. b) The regions of $S_{B}$ (blue), $S_{F}$ (red), and $S_{S}^{\prime}$ (orange) for the same slice.

\section{E. Defining Edge Weights of the Random Walker Graph}

To build the 3D image graph of the constrained Random Walker algorithm, edge weights $w_{i j}^{c r w}$ are defined as:

$$
w_{i j}^{c r w}=1 /\left(1+\beta \cdot\left(g d\left(v_{i}, v_{j}\right)\right)^{2}\right),
$$

where $g d\left(v_{i}, v_{j}\right)$ is the geodesic distance between gray-level intensities of $v_{i}$ and $v_{j}$, and $\beta=3.2$ (see subsection II-F). According to [13], the above function is preferred to the Gaussian function because of its better performance with regard to the quality and stability of the segmentation.

To compute $g d\left(v_{i}, v_{j}\right)$, another graph is built in correspondence to the $3 \mathrm{D}$ image data. In this undirected 26-connected graph, each vertex represents one voxel and two neighboring vertices $\left(v_{i}, v_{j}\right)$ are connected via an edge of weight $w_{i j}^{g d}$. The weights are given by

$$
\begin{aligned}
w_{i j}^{g d} & \left.=\mid\left(g_{i}+\hat{p}_{i}^{\text {mar }}\right)-\left(g_{j}+\hat{p}_{j}^{\text {mar }}\right)\right) \mid \\
& =\left|\left(g_{i}-g_{j}\right)+\left(\hat{p}_{i}^{\text {mar }}-\hat{p}_{j}^{\text {mar }}\right)\right|,
\end{aligned}
$$

where $g_{i}$ is the original (normalized) gray-level intensity of $v_{i}$, and $\left(g_{i}+\hat{p}_{i}^{\text {mar }}\right)$ its augmentation. Considering $\forall v_{i} \in \mathcal{V}$, $\min \left(g_{i}+\hat{p}_{i}^{\text {mar }}\right)$ and $\max \left(g_{i}+\hat{p}_{i}^{\text {mar }}\right)$ are computed and based on these the augmented intensities are normalized to $[0,1]$. 
Then, $g d\left(v_{i}, v_{j}\right)$ is calculated as the smallest sum of the edge weights $w_{m n}^{g d}$ over all possible paths $C_{v_{i}, v_{j}}$ connecting $v_{i}$ and $v_{j}$ through the 26-connected graph. This is given by [14]

$$
g d\left(v_{i}, v_{j}\right)=\min _{C_{v_{i}, v_{j}}} \sum_{m, n} w_{m n}^{g d}, \quad m, n \in C_{v_{i}, v_{j}} .
$$

The above distances are optimally computed in a linear time using an enhanced Dial's algorithm [15]. Having $S_{F}, S_{B}$, $S_{H}, S_{S}$, and $w_{i j}^{c r w}$, the new foreground probability $p_{i}^{c r w}$ is computed by solving the Eq. 3 .
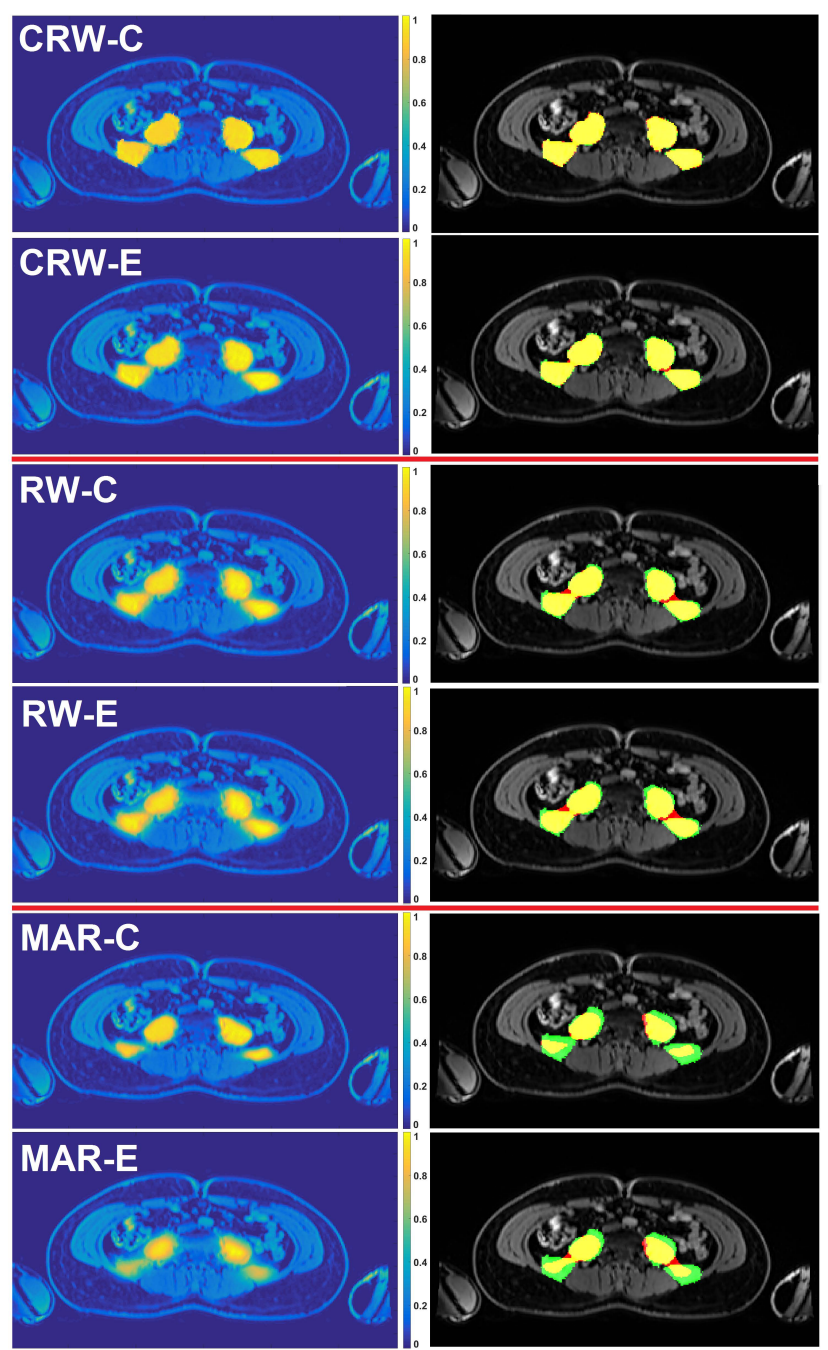

Fig. 5. Left column: An axial slice of the 3D water image of a test subject with normalized intensities of $g_{i}+p_{i}^{\text {crw }}$ (CRW-C), $g_{i}+p_{i}^{\prime c r w}$ (CRW-E), $g_{i}+p_{i}^{r w}$ (RW-C), $g_{i}+p_{i}^{\prime r w}$ (RW-E), $g_{i}+p_{i}^{\text {mar }}$ (MAR-C), $g_{i}+p_{i}^{\prime \text { mar }}$ (MARE). Right column: The corresponding masks of the automatically segmented muscle volumes (red) and the ground truths (green) and their overlap (yellow) over the same slice.

\section{F. Hyperparameter Optimization}

Two hyperparameters, SD of the Gaussian kernel and $\beta$ in Eq. 7, are optimized by a search over all combinations of $\mathrm{SD}=\{1,2, \ldots, 10\}$ and $\beta=\{2,2.2, \ldots, 5\}$ and a 4 -fold cross validation of the automatic segmentation against the ground truths on the training data.

\section{Evaluations ON THE Test Images}

The 3D average and probabilistic muscle atlases are computed using the training data. Then the targeted muscles are automatically segmented over the $3 \mathrm{D}$ water images of $T=28$ test subjects and the segmentation results are evaluated against the manually obtained ground truths $\left\{G_{k}\right\}_{k=1}^{T}$.

This way, the proposed algorithm (CRW) is evaluated against the multi-atlas registration (MAR) and the original Random Walker algorithm (RW) [2] whose labeled seeds are defined in the same way as for the proposed method. These evaluations are done with regard to the segmentation accuracy and its sensitivity to errors in the manually segmented muscle masks used for the training.

To evaluate the aforementioned sensitivity, errors of around $20 \%$ of the muscle volumes are added to the borders of the manually segmented muscle masks of the training data. The resulting erroneous masks are denoted by $\left\{M_{k}^{\prime}\right\}_{k=1}^{M}$. The 3D average and probabilistic muscle atlases are computed once using the correct $\left(\left\{M_{k}\right\}_{k=1}^{M}\right)$ and once using the erroneous $\left(\left\{M_{k}^{\prime}\right\}_{k=1}^{M}\right)$ muscle masks. The resulting correct and erroneous muscle atlases yield muscle probability maps $\left\{P_{k}^{\text {mar }}\right\}_{k=1}^{T}$ and $\left\{P_{k}^{\prime m a r}\right\}_{k=1}^{T}$ for $T=28$ test subjects according to the procedure described in subsection II-D.

The atlas-derived probabilities, contained in $\left\{P_{k}^{\text {mar }}\right\}_{k=1}^{T}$ and $\left\{P_{k}^{\prime \operatorname{mar}}\right\}_{k=1}^{T}$, are also used to determine the labeled seeds, the edge weights, and the constraints of the CRW (see subsections II-D and II-E) and the labeled seeds $\left(S_{F}\right.$ and $\left.S_{B}\right)$ of the RW [2] (in the same way as for the CRW) to eliminate user interactions for seed selection. However, the edges' weights of the RW are defined according to its original proposal, i.e., $w_{i j}^{r w}=\exp \left(-\beta\left(g_{i}-g_{j}\right)^{2}\right)$ [2]. This way, for the CRW, muscle probability maps $\left\{P_{k}^{c r w}\right\}_{k=1}^{T}$ and $\left\{P_{k}^{\prime c r w}\right\}_{k=1}^{T}$, and for the RW, muscle probability maps $\left\{P_{k}^{r w}\right\}_{k=1}^{T}$ and $\left\{P_{k}^{\prime r w}\right\}_{k=1}^{T}$, are obtained. Omitting the methods indicators (CRW, RW, MAR), the automatically segmented muscle masks $\left(\left\{B_{k}\right\}_{k=1}^{T}\right.$ and $\left.\left\{B_{k}^{\prime}\right\}_{k=1}^{T}\right)$ are obtained by extracting the regions of $p_{i} \geq 0.5$ or $p_{i}^{\prime} \geq 0.5$ in those probability maps. Objective evaluations are done by computing the Jaccard index $\left\{J_{k}=\left(B_{k} \cap G_{k}\right) /\left(B_{k} \cup\right.\right.$ $\left.\left.G_{k}\right)\right\}_{k=1}^{T}$ and $\left\{J_{k}^{\prime}=\left(B_{k}^{\prime} \cap G_{k}\right) /\left(B_{k}^{\prime} \cup G_{k}\right)\right\}_{k=1}^{T}$, respectively. Fig. 6 shows the box plots of the computed Jaccard indices for different segmentation methods.

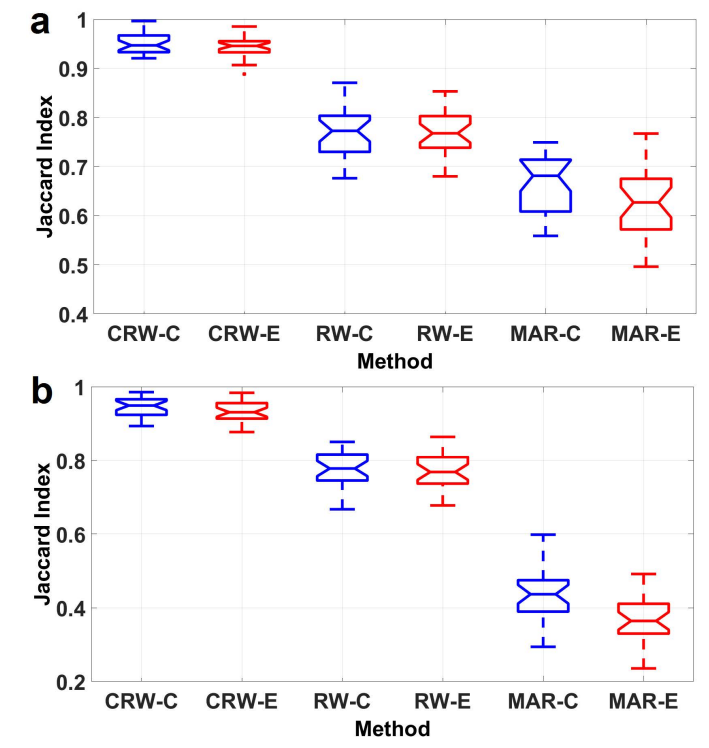

Fig. 6. The Jaccard index comparing the ground truths and the automatically segmented masks of the psoas major (a) and quadratus lumborum (b) muscles obtained from the CRW, the RW, and the MAR using correct (C) or erroneous (E) manual segmentations for atlas computation.

Fig. 5 shows subjective results of the aforementioned 
evaluations for an axial slice of the 3D water image of a test subject. In each case of Fig. 5, the automatic segmentations of the pm and ql muscles are done separately. However, for a more compact demonstration, the automatically segmented masks are merged in one image and compared with the merged ground truths. The CRW using the correct and erroneous muscle atlases are denoted by CRW-C and CRW-E, respectively. Same is done for the RW and the MAR method.

\section{DISCUSSION}

As shown in Fig. 6, MAR has the poorest performance and highest sensitivity to errors in the manually segmented muscle masks used for the training. Its poor performance is due to the small number of training samples. Its sensitivity to the aforementioned errors is due to the direct impact of these errors on final automatic segmentation.

However, our new way of defining edge weights for the Random Walker graph, augmenting intensities of the targeted muscle by a low-pass filtered atlas-derived probability map, and use of additional seeds (constraints) for muscle probability computation lead to the highest performance

Due to the effects of the low-pass filter, the intensity augmentation gets reduced on muscle borders. This, however, does not blur the final segmentation because the random walker defines this segmentation. Also, the employed geodesic distance enhances the delineation of the muscle borders by a better capturing of compositional structures. This is due to the incorporation of both intensity gradients (with respect to the shortest paths) and spatial (Euclidean) distances between intensities in this distance metric [16]. The additional constraints provide a stronger guidance for the graph-based segmentation with regard to the true boundaries of the targeted object $\left(S_{H}\right)$ and the transition between foreground and background regions $\left(S_{S}\right) . S_{H}$ is independent of the muscle atlases and their errors. However, $S_{S}$ varies by such errors but these variations cannot cause a problem for the CRW algorithm as $S_{S}$ can be inside or outside of the muscle.

The performance of the RW and our proposed CRW algorithm has also slightly degraded with errors in the manually segmented masks. This is due to the sensitivity of all RW-based algorithms to the number of labeled seeds [2]-[4], as the foreground seeds get reduced by having errors in the atlas-derived muscle probabilities.

Our way of intensity augmentation (represented in Eq. 9) is similar to an approach proposed for interactive image segmentation [4]. However, instead of histogram-derived priors, we use atlas-derived muscle probabilities to define the edge weights of the random Walker graph. Thus, our approach eliminates the need to estimating the distance between the histograms of the foreground and background intensity distributions in order to appropriately penalize similar intensities of adjacent tissues. This is advantageous because 1) computation of such a distance is expensive, 2) the histogram-based approach cannot improve the segmentation quality if the foreground and background intensity distributions are not well separated. This is the case in many clinical applications since most (healthy) lean tissues show similar intensity distributions on non-contrast-enhanced MR images. This reduces the distance between their histograms and thus boils down the previous approach [4] to the original Random Walker algorithm. We did not address automatic segmentation of pathological tissues. This can be a future extension.

\section{CONCLUSION}

In this paper, we propose a fully automatic approach for $3 \mathrm{D}$ segmentation of muscles on water MR images by tackling challenges due to the similar intensities of adjacent tissues and discontinuities of muscle boundaries. The proposed algorithm uses a small number of training samples and can tolerate errors in the manually segmented muscle masks used for the training. This is achieved by using a constrained Random Walker algorithm with a new automatic mechanism for seed selection and edge weighting.

\section{ACKNOWLEDGMENT}

The MR images of this study are acquired under a financial support from German Research Foundation (DFG) with the grant number of BA 4233/4-1.

\section{REFERENCES}

[1] S. S. Elessawy, M. A. E. Borg, M. A. E. Mohamed, G. E. Elhawary, and E. M. A. El-Salam, "The role of MRI in the evaluation of muscle diseases," The Egyptian Journal of Radiology and Nuclear Medicine, vol. 44, no. 3, pp. 607-615, 2013.

[2] L. Grady, "Random walks for image segmentation," vol. 28, no. 11, pp. 1768-1783, 2006.

[3] — , "Multilabel random walker image segmentation using prior models," in IEEE Computer Society Conference on Computer Vision and Pattern Recognition (CVPR), 2005, pp. 763-770.

[4] W. Yang, J. Cai, J. Zheng, and J. Luo, "User-Friendly interactive image segmentation through unified combinatorial user inputs," vol. 19, no. 9, pp. 2470-2479, 2010.

[5] P. Y. Baudin, N. Azzabou, P. G. Carlier, and N. Paragios, "Prior knowledge, random walks and human skeletal muscle segmentation," in 15th International Conference on Medical Image Computing and Computer-Assisted Intervention (MICCAI), 2012, pp. 569-576.

[6] C. M. Engstrom, J. Fripp, V. Jurcak, D. G. Walker, O. Salvado, and S. Crozier, "Segmentation of the quadratus lumborum muscle using statistical shape modeling," J Magn Reson Imaging, vol. 33, no. 6, pp. 1422-1429, 2011.

[7] A. Karlsson, J. Rosander, T. Romu, J. Tallberg, A. Groenqvist, M. Borga, and O. Dahlqvist Leinhard, "Automatic and quantitative assessment of regional muscle volume by multi-atlas segmentation using whole-body water-fat MRI," J Magn Reson Imaging, vol. 41, no. 6, pp. 1558-1569, 2015.

[8] S. Andrews and G. Hamarneh, "The generalized log-ratio transformation: Learning shape and adjacency priors for simultaneous thigh muscle segmentation," IEEE Trans Med Imaging, vol. 34, no. 9, pp. 1773-1787, 2015.

[9] J. Canny, "A computational approach to edge detection," vol. PAMI-8, no. 6, pp. 679-698, 1986.

[10] S. Ourselin, A. Roche, G. Subsol, X. Pennec, and N. Ayache, "Reconstructing a 3D structure from serial histological sections," Image and Vision Computing, vol. 19, pp. 25-31, 2001.

[11] D. Forsberg, M. Andersson, and H. Knutsson, "Parallel scales for more accurate displacement estimation in phase-based image registration," in International Conference on Pattern Recognition, 2010, pp. 2329-2332.

[12] _ - International Conference on Image Analysis and Recognition, 2012, ch. Non-rigid diffeomorphic image registration of medical images using polynomial expansion, pp. 304-312.

[13] L. Grady and M.-P. Jolly, "Weights and topology: A study of the effects of graph construction on 3d image segmentation," in Proceedings of the 11th International Conference on Medical Image Computing and Computer-Assisted Intervention, 2008, pp. 153-161.

[14] X. Bai and G. Sapiro, "Geodesic matting: A framework for fast interactive image and video segmentation and matting," International Journal of Computer Vision, vol. 82, no. 2, pp. 113-132, 2009.

[15] L. Yatziv, A. Bartesaghi, and G. Sapiro, "O(N) implementation of the fast marching algorithm," J Comput Phys, vol. 212, no. 2, pp. 393-399, 2006.

[16] A. Criminisi, T. Sharp, and A. Blake, GeoS: Geodesic Image Segmentation, 2008, pp. 99-112. 